\title{
Huellas afectivas del trabajo en política de protección a la infancia
}

\author{
Javiera Garcia-Meneses, Mg. ${ }^{\text {a }}$ \\ Pontificia Universidad Católica de Valparaíso, Chile \\ Iván Chanez-Cortés ${ }^{b}$ \\ Pontificia Universidad Católica de Valparaíso, Chile
}

\author{
Carla Fardella-Cisternas, Ph. D. ${ }^{\mathrm{c}}$ \\ Universidad Andrés Bello, Chile
}

Alejandra Corvalán-Navia, Mg. ${ }^{\mathrm{d}}$

Universidad Andrés Bello, Chile

javiera.garcia.m@mail.pucv.cl

\section{Resumen (analítico)}

Chile es un caso emblemático en la instalación de una política de protección a la infancia con orientación neoliberal. Actualmente, esta política es ejecutada casi completamente por organizaciones privadas, financiadas conforme al logro de indicadores de desempeño estandarizados. Algunas investigaciones plantean que estos indicadores gobiernan la racionalidad de los trabajadores y trabajadoras; empero, estudios internacionales señalan que este tipo de gobernanza no solo es racional, sino también afectiva. A partir de un análisis textual-afectivo de 14 entrevistas individuales y dos talleres grupales realizados a cinco trabajadoras y un trabajador de distintos organismos colaboradores del Servicio Nacional de Menores, se reporta que este modelo de gestión consume y maquiniza los cuerpos de estas y estos profesionales. No obstante, el afecto es el que hace posible la resistencia.

\section{Palabras clave}

Afecto; bienestar de la infancia; neoliberalismo; nueva gestión pública; política social; subjetividad laboral.

\section{Thesauro}

Tesauro de Ciencias Sociales de la Unesco.

\section{Para citar este artículo}

Garcia-Meneses, J., Chanez-Cortés, I., FardellaCisternas, C., \& Corvalán-Navia, A. (2022). Huellas afectivas del trabajo en política de protección a la infancia. Revista Latinoamericana de Ciencias Sociales, Niñez y Juventud, 20(1), 1-22. https://dx.doi.org/10.11600/rlcsnj.20.1.5144

\section{Historial}

Recibido: 02.06.2021

Aceptado: 01.10.2021

Publicado: 14.12.2021

\section{Información artículo}

Este artículo deriva de la investigación Construcción subjetiva del trabajador de la política social en Chile: una aproximación desde las prácticas afectivas y presenta los resultados del trabajo desarrollado entre mayo y septiembre de 2020. El estudio cuenta con el apoyo financiero de la Agencia Nacional de Investigación y Desarrollo, Programa de becas Doctorado Nacional, 2020, 21202092; del Fondo Nacional para el Desarrollo de la Ciencia y la Tecnología (Proyecto 1191015); y del Centro Núcleo Milenio Autoridad y Asimetrías de Poder (NCS17_007). Área: psicología. Subárea: psicología social del trabajo. 


\section{Affectivity in the implementation of child welfare policies}

\section{Abstract (analytical)}

Chile is an emblematic case in the implementation of a neoliberal child welfare policy. This policy is now almost entirely undertaken by private organizations, financed based on their achievement of standardized performance indicators. Scientific literature suggests that these indicators govern workers' rationality. However, international studies indicate that the effect of indicators is not just rational but also affective. From a textual-affective analysis of 14 interviews and 2 workshops conducted with 5 female workers and 1 male worker from different partner organizations within the Chilean National Service for Minors, participants reported that this management model consumes and mechanizes the work carried out by these professionals. However, it is affectivity that makes resistance possible.

Keywords

Affect; child welfare services; neoliberalism; new public management; social policy; work subjectivity.

\section{Traços afetivos do trabalho de política de proteção à infância}

\section{Resumo (analítico)}

O Chile é um caso emblemático na instalação de uma política neoliberal de proteção à criança. Esta, é hoje quase inteiramente executado por organizações privadas, financiadas de acordo com a obtenção de indicadores de desempenho padronizados. Pesquisas sugerem que estes indicadores governam a racionalidade dos trabalhadores; enquanto estudos internacionais indicam que este governo não é apenas racional, mas também afetivo. A partir de uma análise textual-afetiva de 14 entrevistas e duas oficinas realizadas a cinco trabalhadoras e um trabalhador de diferentes Organizações Colaboradoras do Serviço Nacional de Menores, é relatado que este modelo de gestão consome e mecaniza os órgãos destes profissionais. No entanto, é o afeto que torna possível a resistência.

\section{Palavras-chave}

Afeto; serviços de bem-estar infantil; neoliberalismo; nova gestão pública; política social; subjetividade laboral.

\section{Información autores}

[a] Psicóloga y candidata a doctora en Psicología, Pontificia Universidad Católica de Valparaíso (Chile). (iD) 0000-0002-4470-4427. H5: 1. Correo electrónico: javiera.garcia.m@mail.pucv.cl

[b] Psicólogo, Pontificia Universidad Católica de Valparaíso (Chile). iD 0000-0002-8737-179X. H5: 0. Correo electrónico: ivanchanez@gmail.com

[c] Doctora en Psicología Social, Universitat Autónoma de Barcelona (España). iD 0000-0001-8936-2435. H5:

13. Correo electrónico: carla.fardella@unab.cl

[d] Magíster en Análisis y Conducción de Grupos, Universitat de Barcelona (España). Candidata a doctora en Educación y Sociedad, Universidad Andrés Bello (Chile). iD 0000-0002-6329-403X. H5: 1. Correo electrónico: alejandracorvalan@gmail.com 


\section{Introducción}

A nivel internacional, Chile es considerado como un caso emblemático en la et al., 2021). El neoliberalismo ha sido definido como una forma de gobierno económico y social que ha transformado la relación entre el Estado, el mercado y la sociedad civil (Pavez et al., 2019). En el caso particular chileno, desde el inicio del periodo de la Dictadura militar en 1973 se han realizado una serie de reformas neoliberales estructurales caracterizadas por rechazar el rol intervencionista del Estado, disminuir la planificación estatal, reducir los mecanismos de protección social de los ciudadanos y las ciudadanas, disminuir las regulaciones del mercado laboral y privatizar los servicios públicos (Araujo, 2019; Cubillos-Celis, 2021).

Junto con la incorporación y el fortalecimiento del neoliberalismo como modelo económico, se instaló el nuevo management público (en adelante NMP) como forma de organización del sector público (Hood, 1991). Esta nueva lógica de gestión (promovida por organismos internacionales como el Fondo Monetario Internacional y la Organización para la Cooperación y el Desarrollo Económico) se ha convertido en una práctica central en la gestión de los servicios públicos en los países con modelos económicos neoliberales (Organisation for Economic Co-operation and Development, 2005). El NMP busca hacer a los Estados similares a una empresa, con el fin de lograr estándares de eficiencia y eficacia que sean rentables económicamente (Soto et al., 2016). En Chile, la incorporación del NMP implicó la privatización de los servicios sociales y el estímulo a la competencia entre los prestadores de servicios a través de procesos de licitación pública y contratación temporal; todo bajo un sistema de financiamiento en función del cumplimiento de objetivos e indicadores de desempeño cuantificables (Pereira, 2015).

La incorporación del NMP ha reorganizado la provisión de diversos servicios tales como la educación, la salud y las políticas sociales (Vivero-Arriagada, 2017). Ejemplo de ello es la política de protección a la infancia chilena - parte de las políticas sociales-, 
actualmente ejecutada en más de un $95 \%$ por organizaciones privadas denominadas organismos colaboradores acreditados (Servicio Nacional de Menores, 2019). La privatización de esta política ha implicado que dichos organismos deban ofrecer sus servicios de acuerdo con las propuestas desarrolladas por el Estado y por el Servicio Nacional de Menores (Sename), compitiendo entre sí cada dos o tres años, a través de procesos de licitación pública, para asegurar su financiamiento (Pereira, 2015). En caso que estas organizaciones logren adjudicarse los proyectos, la entrega del financiamiento está condicionada al logro de una serie de metas cuantificables (Rojas, 2018). Estas metas son el cumplimiento de un número mínimo de atenciones mensuales por niño, niña o adolescente (en adelante NNA) ingresado en las organizaciones, la sistematización de cada intervención realizada por los y las profesionales, el desarrollo de informes de avance trimestrales, entre otros. Estos indicadores medirán el desempeño de las organizaciones y su logro asegurará tanto el depósito íntegro de la subvención, así como también la adjudicación de futuras licitaciones (Varas et al., 2018).

La instalación de sistemas de competencia y financiamiento según resultados como formas de gestión de la política protección de la infancia ha afectado profundamente la construcción subjetiva de sus trabajadores y trabajadoras (Albaek et al., 2018). Respecto a ello, la literatura especializada nacional e internacional ha planteado que los nuevos mecanismos de gestión incorporados al sector público son formas de modelamiento de racionalidades (Fardella-Cisternas et al., 2021; Marques \& Dal Castel, 2015; Sisto, 2020; Soto et al., 2016). Según estas investigaciones, estos mecanismos de gestión son dispositivos de gobierno (Foucault, 2008; Sisto \& Zelaya, 2013) que han reducido la función pública a mediciones estandarizadas y resultados prescritos con el propósito de regularizar y convertir la práctica profesional en objetivos estrechamente definidos y medibles; así, alineando las subjetividades laborales de estas y estos profesionales en torno a la figura del trabajador público eficiente: un individuo orientado únicamente a cumplir las metas establecidas por el Estado (Hunter, 2015).

No obstante, en los últimos años, diversos estudios (Errázuriz, 2021; Glinsner et al., 2019; Jupp et al., 2017; McKenzie, 2017; Penz \& Sauer, 2019; Rojas, 2018) han señalado que el gobierno de las trabajadoras y los trabajadores no es únicamente racional, sino que también es afectivo. Respecto a esto, estudios internacionales enmarcados en las teorías afectivas poshumanistas (Ashcraft, 2017; Katila et al., 2017; Nishida, 2017; Penz et al., 2017; Pitton \& McKenzie, 2020) convergen en que la incorporación del NMP implicaría 
nuevos modos de gobernanza, los cuales estarían mediados por las relaciones afectivas entre la política de infancia, sus ejecutoras y ejecutores, así como sus usuarias y usuarios.

A modo general, los afectos han sido definidos como intensidades preconscientes que incrementan o decrecen las capacidades agenciales y existenciales de los cuerpos que se encuentran en una relación (Seigworth \& Gregg, 2010). Para las teorías afectivas, la producción social emerge del ensamblaje entre cuerpos (humanos y no-humanos) que se afectan entre sí, y de las consecuentes capacidades que derivan de estas relaciones (Fox \& Alldred, 2013). Llevado al trabajo en política de infancia, esto implica, por ejemplo, que las organizaciones que ejecutan la política son producidas a partir del complejo entramado afectivo que emerge de la relación cotidiana entre los cuerpos-humanos y no-humanos que las constituyen (Gherardi \& Laasch, 2021).

Respecto al concepto de cuerpo, para las teorías afectivas un cuerpo es todo aquello que tiene la capacidad de afectar y ser afectado (Seigworth \& Gregg, 2010). En este sentido, cuando se habla de cuerpos que se encuentran en una relación, se considera a la política de protección a la infancia misma, a los sistemas de financiamiento según resultados y a los indicadores de desempeño como cuerpos no-humanos que tienen la capacidad de participar en el paso de los afectos (Gherardi et al., 2018). Como señalan diversos estudios (Errázuriz, 2021; Fox \& Alldred, 2013; Gherardi \& Laasch, 2021; Shöngut-Grollmus, 2017), en la repetición cotidiana de estas relaciones afectivas, los cuerpos humanos y nohumanos que constituyen la política de infancia se ensamblan entre sí, afectándose mutuamente. Mediante y en este ensamblaje los afectos circulan entre los cuerpos, produciendo capacidades específicas de acción que pueden (re)producir las maneras en las que se ejecuta la política de protección a la infancia en el espacio local o (trans)formar las maneras en las que trabajadores y trabajadoras experimentan la cotidianidad de su trabajo (Ahmed, 2017).

Mientras los indicadores de evaluación y desempeño del NMP pueden ser pensados como abstracciones sin vida que controlan a los trabajadores y trabajadoras, la perspectiva propuesta por la teoría del afecto nos permite comprenderlos como cuerpos no-humanos que tienen la capacidad de afectar la experiencia de los diferentes sujetos involucrados en una relación (McKenzie, 2017). Así, este artículo busca responder la pregunta: ¿cómo los trabajadores y las trabajadoras de la política de protección a la infancia en Chile son interpelados afectivamente por los sistemas de rendición de cuentas, los indicadores de evaluación de desempeño y otros elementos que componen dicha política? Desde ahí, el objetivo de este estudio es conocer los afectos que se mueven entre los 
cuerpos que constituyen la política de infancia y comprender el impacto de estos en la experiencia laboral de los trabajadores y las trabajadoras. Los resultados presentados en este artículo permiten conocer las huellas afectivas que los instrumentos de gestión neoliberal dejan en los cuerpos de trabajadores y trabajadoras. Estos hallazgos aportan un nuevo lente analítico mediante el cual es posible teorizar el ensamblaje entre estos dispositivos de gestión y la construcción subjetiva de la fuerza de trabajo de la política de protección a la infancia chilena.

\section{Método}

El propósito de esta investigación fue comprender cómo los trabajadores y las trabajadoras de la política de protección a la infancia en Chile experimentan las interpelaciones afectivas de los dispositivos de control de gestión propuestos por el Estado a través de Sename. Para esto se optó por un enfoque metodológico cualitativo de carácter local, específico y contextual (Flick, 2004) y se realizó un diseño etnográfico digital (Pink et al., 2016).

La etnografía digital tuvo una duración de tres meses (julio-septiembre 2020) y participaron cinco trabajadoras y un trabajador (tabla 1 ) pertenecientes a distintos organismos colaboradores acreditados por el Sename. El contacto con el trabajador y las trabajadoras se realizó mediante el Sindicato Interempresa Nacional de Trabajadores/as Subcontratados del Servicio Nacional de Menores. La selección de las personas participantes se realizó a través de un muestreo orientado por la información (Flyvbjerg, 2011), cuyos criterios de selección consideraron el tipo de programa (es decir, que fuesen programas de la línea ambulatoria de Sename) y la jerarquía de las personas trabajadoras (que no tuviesen cargos de jefatura). En relación con las características sociodemográficas de las personas seleccionadas, todas pertenecían a organizaciones no gubernamentales ubicadas en la región de Valparaíso, Chile. La edad promedio de ellas fue de 31 años y su tiempo de ejercicio profesional en la red Sename fluctuó entre los 1 y 8 años.

Respecto a la producción de datos, a lo largo del proceso etnográfico se realizaron dos rondas de entrevistas activas virtuales (Denzin, 2001), con el objetivo de abarcar las experiencias singulares de cada participante (Holstein \& Gubrium, 1995). Estas entrevistas estuvieron orientadas a conocer la experiencia laboral y las prácticas cotidianas de trabajo de este y estas profesionales. Asimismo, se realizaron dos talleres de reflexión grupales guiados por el método de biografía colectiva (Davies \& Gannon, 2006), donde 
se profundizó sobre los afectos presentes en el quehacer cotidiano. Cabe destacar que todo el proceso fue realizado mediante la plataforma Zoom debido a la contingencia sanitaria generada por el COVID-19.

\section{Tabla 1}

Numeración de documentos y descripción de los y las participantes del estudio

\begin{tabular}{|c|c|c|c|}
\hline n. ${ }^{\circ}$ documento & Profesión & $\begin{array}{l}\text { Años de ejercicio } \\
\text { profesional en Sename }\end{array}$ & $\begin{array}{l}\text { Programa al que pertenece } \\
\text { actualmente }\end{array}$ \\
\hline D1 (grupal 1) & - & - & - \\
\hline D2; D12 & Socióloga & & Residencia \\
\hline D3; D14 & Psicóloga & 7 años & Programa reparatorio en maltrato \\
\hline D4; D13 & Psicólogo & 7 años & Programa de intervención especializada \\
\hline D5 (piloto) & Psicóloga & 3 años & Programa de prevención focalizada \\
\hline D6 (piloto) & Técnico asistente social & 1 año & Programa de prevención focalizada \\
\hline D7 (grupal 2) & - & - & - \\
\hline D8; D15 & Trabajadora social & 4 años & Programa de prevención focalizada \\
\hline D9; D11 & Trabajadora social & 8 años & Familias de acogida especializada \\
\hline D10; D16 & Secretaria administrativa & 7 años & Programa reparatorio en maltrato \\
\hline
\end{tabular}

Nota. Los documentos donde no se señala la profesión o programa corresponden a las transcripciones de los talleres grupales.

El diseño de esta investigación fue aprobado por el Comité de Bioética y Bioseguridad de la Pontificia Universidad Católica de Valparaíso, Chile. En cada una de las instancias de producción de datos, se entregó a el y las participantes un consentimiento informado que contenía la descripción y objetivo de las actividades, así como los aspectos éticos y de confidencialidad de la investigación. Este documento debió ser firmado antes de comenzar con las actividades ya mencionadas. Para garantizar la integridad ética de la investigación, todos los nombres de las personas participantes y de las organizaciones en las que estas trabajaban fueron anonimizadas.

El análisis de los datos se realizó, en un primer momento, a través de la codificación in vivo propuesta por la práctica de la teoría fundamentada (Charmaz, 2007). Esta técnica contribuyó a la simplificación de los datos, identificando relatos particulares y agrupándolos en códigos más pequeños. De este análisis, se obtuvieron 75 códigos que fueron analizados en mayor profundidad mediante un análisis textual-afectivo (Cromby, 2012), el cual considera que experiencia y lenguaje están mutuamente interpenetrados. En este 
análisis, el lenguaje no fue analizado en torno a su significado, sino que el foco estuvo en estudiar las palabras en relación con la materialidad y sensorialidad de los cuerpos (Enciso, 2015), considerando que la experiencia narrada y los significados de esta descansan y se constituyen a través del cuerpo (Ruthrof, 1997). El procedimiento del análisis afectivo estuvo orientado por dos preguntas: la primera, aludió al afecto como una dinámica relacional, preguntándose: ¿qué cuerpos humanos o no-humanos se relacionan o ensamblan entre sí? La segunda, buscó profundizar en ¿qué afectos se mueven y cómo se experimentan en los cuerpos humanos?

\section{Resultados}

A continuación, reportamos los principales resultados sobre cómo los trabajadores y trabajadoras experimentan las interpelaciones afectivas de los instrumentos de gestión del NMP presentes en la política de protección a la infancia chilena. Los resultados fueron organizados en tres categorías: trabajo administrativo, política de protección a la infancia y política de infancia imaginada.

\section{Trabajo administrativo: complicado, devastador e indiferente}

Según los relatos, una de las características más evidentes del trabajo en programas que ejecutan la política de protección a la infancia en Chile es la sobrecarga laboral. Esta es explicada por el y las profesionales en relación con el trabajo administrativo que deben realizar cotidianamente. En concreto, el trabajo administrativo implica sistematizar (en digital o papel) los procesos de intervención y cada una de las actividades que se realizan con los NNA, ya sea: 1) en la plataforma virtual del Servicio Nacional de Menores; 2) en la carpeta física de seguimiento de los casos de cada organización; 3) los informes de permanencia de los programas; 4) los informes al Juzgado de familia; 5) la actualización de los planes de intervención, entre otras cosas ( $\left.\mathrm{D}_{5}, 16\right){ }^{1}$

El trabajo administrativo es definido por las personas trabajadoras como complicado, devastador e indiferente. Es complicado debido a que está compuesto de muchos aspectos muy específicos e intrincados que dificultan su comprensión. Uno de tales aspectos es que, a pesar que dentro de los programas hay familias, el trabajo de sistematización de

\footnotetext{
${ }^{1}$ Los fragmentos de las entrevistas están citados de la siguiente forma: número de documento, número de cita de ATLAS.ti.
} 
las intervenciones debe realizarse por cada uno de los NNA, independiente de sus relaciones de parentesco. Esto se vincula directamente con el hecho que la política de protección a la infancia en Chile es una política focalizada de intervención que está diseñada para operar sobre problemas sociales específicos, dirigidos a un cierto tipo de usuario de manera individual (Schöngut-Grollmus, 2017). Como menciona esta entrevistada:

Yo por ejemplo ahora tengo... cuatro hermanos y que son tres causas distintas (...); es el mismo tribunal, están viendo lo mismo, los cuatro cabros ${ }^{2}$ viven en la misma casa, con el mismo cuidador (...), pero así tengo que ir informando. (D11, 62)

Por otro lado, el trabajo administrativo es construido como devastador, puesto que destruye el sentido de tareas centrales del trabajo de protección de derechos de infancia, como por ejemplo, la intervención. Este tipo de trabajo es definido como un cuerpo que pesa más que otros cuerpos, por lo que, a su paso, arrasa con el sentido y las formas particulares de ejecutar ciertas acciones, reconstruyéndolas a su modo. Como menciona el entrevistado: «pesa más la burocracia, pesa más la formalidad y, finalmente, terminamos tratando a los [NNA] que no están judicializados como si lo estuvieran [cursivas añadidas]» $\left(\mathrm{D}_{4}, 8\right)$.

Por último, el trabajo administrativo es percibido como indiferente, en tanto no le interesa relacionarse con las personas ni mostrarse atento a sus necesidades. Esta aspereza del trabajo administrativo afecta directamente el proceso de intervención, vaciándolo de su sentido ético, es decir, de su orientación hacia el bienestar de los NNA a través del establecimiento de una relación local, vincular y afectiva (Fardella-Cisternas et al., 2016).

Yo de hecho recuerdo que teníamos una tabla de Excel donde marcamos a quién habíamos visto; y ya finalmente importaba más marcar el cuadrito de Excel y decir que los vi, a efectivamente preguntarle algo o saber cómo estaba. $\left(\mathrm{D}_{4}, 50\right)$

Estas características del trabajo administrativo afectan a los cuerpos de trabajadores y trabajadoras, generando la sensación de terror y de falta de energía. El terror que genera el trabajo administrativo se relaciona con que el cumplimiento de las labores administrativas tributa directamente al financiamiento de las organizaciones. El logro de las metas establecidas por Sename debe ser respaldado mediante procesos mensuales de rendición de cuentas que aseguran el pago íntegro de la subvención a las organizaciones (Pavez,

\footnotetext{
${ }^{2}$ Chilenismo para niños, niñas o adolescentes.
} 
2018). Uno de los aspectos relevantes de este proceso es que, si bien la rendición es realizada por la organización, la responsabilidad del funcionamiento correcto del proceso recae en cada trabajador y trabajadora de manera individual.

Por ejemplo, te avisan que van a ir a hacer una supervisión; entonces te dicen: «Oye, van a revisar todas las carpetas de los 80 niños» (...); entonces tienes que empezar a revisar las carpetas, los cabros [trabajadores y trabajadoras] se desesperan porque los tienen escritos en el computador y no lo imprimieron y si no está en la carpeta no lo pagan, y empieza la semana horrible. Entonces esa es la campaña del terror. (D16, 29)

Por otro lado, la sensación de falta de energía se relaciona con que estos trabajadores y trabajadoras experimentan el trabajo administrativo como algo que les quita el vigor. Esto se explica cuando consideramos que este tipo de trabajo no tiene sentido dentro de su quehacer laboral cotidiano y, sin embargo, es la labor que ocupa la mayor parte de su tiempo. Este sinsentido es tal que cuando las personas entrevistadas se refieren al cumplimiento de labores administrativas generalmente son descritas como «hacer nada» $(D 16,67)$. Así, el exceso de trabajo administrativo no es reconocido como un trabajo valioso dentro de las labores cotidianas, lo cual hace que estos trabajadores y estas trabajadoras gasten las fuerzas que les quedan en realizar más y mejores intervenciones para reatribuirle el sentido a su trabajo, haciendo que «el cansancio sea interminable» (D14, 2; D16, 31) y «haga imposible la resistencia» (D15, 45).

En síntesis, el trabajo administrativo es percibido como un cuerpo que se caracteriza por ser complicado, devastador e indiferente. Estas características generan en los trabajadores y las trabajadoras una sensación de terror y falta de energía que las mantiene en un sentido de constante crisis (Mckenzie, 2017), las consume y les impide resistirse a los embates de la sobrecarga laboral administrativa (Gómez-Villar, 2016). Será esta construcción del trabajo administrativo y la experiencia afectiva que tienen los trabajadores y las trabajadoras en relación con este, lo que permita el disciplinamiento de aquellos cuerpos abyectos que no se alineen a la ejecución neoliberal de la política de protección a la infancia (Bealer, 2019).

\section{Política de protección a la infancia: empresa deshumanizadora}

Acorde con lo señalado por las personas entrevistadas, los organismos colaboradores acreditados de Sename para los cuales trabajan se organizan de manera similar a cómo lo 
hace una empresa que vende productos. La razón de esto es que la ejecución de la política está orientada primordialmente a lo cuantitativo, olvidando la complejidad de los procesos de intervención y los límites de las capacidades corporales y emocionales de los trabajadores y las trabajadoras (Pavez et al., 2019).

Las ONG en este caso, que son las empresas privadas que se adjudican esos proyectos, lo que hacen es tratar de abaratar costos (...). Ese fenómeno es súper de empresa privada, al fin y al cabo, que es como tratar de darle una respuesta a un problema por encima, solamente para poder llegar a las metas que me propone el servicio, pero no le estoy dando un énfasis a la complejidad del Sename. $($ D8, 39)

El hecho que las organizaciones que ejecutan la política de protección a la infancia funcionen análogamente a una empresa privada que vende mercancías, ha construido un cuerpo ideal de trabajador o trabajadora que se caracteriza por ser un cuerpo masculino, que no tiene a su cargo el cuidado de personas, por lo que puede preocuparse solo de su trabajo y que, a su vez, tiene ahorros suficientes para no preocuparse por el hecho de percibir bajas remuneraciones $\left(\mathrm{D}_{2}, 25 ; \mathrm{D}_{5}, 54 ; \mathrm{D}_{16}, 47\right)$. Asimismo, es un trabajador que no cuestiona el modelo y no es reflexivo, de manera tal que cumple todo lo que se le solicita (D4, 53; D6, 48; D16, 26). Es técnico y calculador, es decir, no se ve implicado en lo que les sucede a los NNA y sus familias $\left(\mathrm{D}_{5}, 54 ; \mathrm{D}_{14}, 10\right)$ y es capaz de organizar todo prolijamente, independiente de si los usuarios o usuarias no quisieron contarle sus historias en profundidad, porque, para él, lo importante es que los informes estén completos en el tiempo estipulado ( $\left.\mathrm{D}_{3}, 109 ; \mathrm{D} 8,81 ; \mathrm{D} 9,58\right)$.

Esta construcción de trabajador ideal ha afectado la experiencia laboral cotidiana de los trabajadores y las trabajadoras. Respecto a esto, estas señalan que se sienten como una máquina o como una pieza u objeto completamente remplazable. En cuanto a la metáfora de la máquina, esta se refiere a la sensación que trabajadores y trabajadoras pueden ser programados para producir intervenciones de la misma manera en la que una máquina es programada para ensamblar productos. Como las máquinas, estos y estas profesionales no tienen ni consciencia ni emociones, por lo cual no es necesario preguntarles su opinión ni sus sentimientos, siendo su fin último el aumento de la productividad de la empresa al menor costo y en el menor tiempo posible. Como menciona esta entrevistada:

Entrevistador: ¿Cómo dirías tú que ve la política de infancia a los trabajadores y a las trabajadoras? 
Entrevistada: Como máquinas (...). No se ve que el trabajador también es persona y que tiene límites; tiene el límite que le permite su cuerpo, su humanidad (...). A lo mejor uno con las máquinas uno puede doblar 200 cajas en una hora; y es como que el interventor puede hacer 24 sesiones a la semana sin pensar; por ejemplo, que a veces uno también tiene un impacto emocional que va a enlentecerla (sic), o a veces uno tiene problemas en la casa, o a veces con las mismas familias. (D14, 25)

Asimismo, las personas entrevistadas señalan que la política de protección a la infancia las hace sentir como piezas movibles o reemplazables. Con esto se refieren a que sienten que esta política los y las ve como trabajadores vacíos, sin contenido. En este sentido, los cuerpos de los trabajadores y las trabajadoras son objetivados y despojados de su humanidad (Chen, 2012), pasando a ser un cuerpo insignificante e inanimado, que se puede mover y desechar sin ningún cambio sustancial en la maquinaria productiva.

La metáfora de la pieza reemplazable se ve con claridad cuando las personas entrevistadas hablan de la alta rotación de personal que hay dentro de las organizaciones, la cual está permitida por los convenios entre estas y Sename. Ejemplo de ello es lo que ocurre en los periodos de licitación, en donde se finalizan los contratos y se cambian a los trabajadores y las trabajadoras de un programa a otro sin mayor importancia, bajo la excusa de «todos hacen lo mismo»:

Normalmente se tiene muy poco en cuenta (...) de que sea un profesional que tenga más experiencia (...) y eso en el fondo se nota cuando hacemos licitaciones cada dos años y hay que despedir a todos y cambiarse y es como que diera lo mismo..., yo Mariela y después pasa Juanita; "jah! da lo mismo, porque es otra trabajadora social, que va a hacer la misma pega, exactamente lo mismo»... Entonces, eres un trabajador social como sin..., sin contenido, insignificante no más..., vacío..., que se puede despedir, recontratar, mover y no importa. (D11, 21)

Una de las implicaciones de que los cuerpos de los trabajadores y trabajadoras sean considerados como cuerpos-máquina u cuerpos-objeto es la transformación de los procesos de intervención psicosocial y las maneras en las que se perciben los cuerpos de los NNA que participan de ella. En este sentido, si los cuerpos de las personas que trabajan en la política de infancia son cuerpos-máquina, los NNA se transforman en productos. En concordancia, los NNA son vistos por la política «como objetos que puedo reparar...; son objetos que, bueno vienen porque están súper fisurados, súper fragmentados» $\left(\mathrm{D}_{16} 6,5_{1}\right)$. 
Sumado a esto, como menciona una de las entrevistadas, la intervención en la política de infancia se transforma en «un servicio técnico, como un centro de reparación» (D14, 28).

En resumen, los organismos colaboradores acreditados de Sename funcionan de manera similar a una empresa privada que produce manufacturas para su posterior comercialización. La orientación de esta política hacia lo cuantitativo, por sobre los procesos personales de los trabajadores, las trabajadoras y los NNA, interpela a estos cuerpos de manera tal que los deshumaniza. Así, esta política ha transformado a los cuerpos de trabajadores y trabajadoras en cuerpos-máquina; es decir, cuerpos no-humanos cuya función principal es producir. Esta deshumanización ha tenido un impacto en las maneras de concebir la intervención y a los NNA: esta se ha transformado en un proceso estandarizado de reparación, mientras que los NNA se han transformado en productos dañados, que deben ser recompuestos en un corto periodo de tiempo.

\section{Política de infancia imaginada: rehumanizadora y agencial}

En los relatos emerge como una temática recurrente el deseo de una política de protección a la infancia diferente. Para las personas entrevistadas la ideal sería: universal $\left(D_{13}, 46\right)$, tendría una relevancia primordial entre las políticas sociales (D11, 56), contemplaría un enfoque de derechos (D12, 54), consideraría la complejidad de las intervenciones y buscaría articular las diferentes políticas de desarrollo social (D16, 77), comprendiendo que los problemas de la sociedad son parte un sistema que no puede ser fragmentado (D15, 53).

Esta política ideal de protección a la infancia se convertirá en un cuerpo imaginado (Ahmed, 2017) que afectará la relación que tienen estos trabajadores y trabajadoras con la política social que tiene hoy una existencia material —en términos de leyes, planes y programas- en Chile. Esta política imaginada transformaría a estos y estas profesionales en agentes, moviéndolos a rechazar los embates de la política existente.

Entrevistador: ¿Cómo [esta política ideal] cambia la metáfora de trabajador que me dijiste en un inicio? La idea de este trabajador máquina.

Entrevistado: Uy..., yo creo que es como..., de ser como agente; algo así como..., como agente po.' $\left(\mathrm{D}_{15}, 5 \mathrm{c}\right)$

${ }^{3}$ Chilenismo; pues. 
En este sentido, la política imaginada-ideal afecta las capacidades de los cuerpos de los trabajadores y trabajadoras devolviéndoles a una posición de agentes, capaces de transformar los contextos en los que ejecutan su labor. Esta nueva capacidad de acción transforma las maneras en las que estos trabajadores y trabajadoras se describen en relación con su trabajo, surgiendo metáforas de sí relacionadas con seres vivos, lugares con movimiento orgánico u objetos que permiten morar en lugares profundos, como, por ejemplo: un canal (D14, 13), un batiscafo que alumbra las profundidades del mar (D13, 38) o un ave fénix (D16, 6o). Así, trabajadores y trabajadoras se redefinen como cuerpos que sienten y viven, volviendo a tener forma humana, desmaquinizándose.

Yo creo que [la metáfora de mí como trabajadora] es eso, como de tender la mano o tener los brazos abiertos; no solamente para ayudar o acoger, sino como ese encuentro humano, donde uno a veces, claro, tiene que sostener algunas cosas; o cuando hay situaciones complejas donde alguno a veces tiene que tender la mano o a veces uno tiene que estrechar la mano de otro. Como esto de acordar cosas, de saludarse, de encontrarse (...). Es al revés a esto de que uno es máquina o se considera máquina. $\left(\mathrm{D}_{14}, 35\right)$

Esta rehumanización y valoración del cuerpo de los trabajadores y trabajadoras los despierta a la reflexión, la cual mueve estos cuerpos a la resistencia. Esta resistencia es entendida como una forma de subvertir la política de infancia, así como también la capacidad de soportar aquello que oprime. En otras palabras, la resistencia es una fuerza que empuja a otros cuerpos al cambio; así como también una fuerza que los presiona. Sumado a esto, es importante resaltar que en los relatos la resistencia no es solo una acción que estos y estas profesionales ejecutan diariamente, sino que es un elemento constitutivo de sus cuerpos: estos son resistencia.

Entrevistador: ¿Cómo definirías tú a los trabajadores y a las trabajadoras?

Entrevistado: En una palabra: resistencia. Yo creo que eso es resistencia (...). Yo creo que al principio uno interioriza todo, pero después viene como un despertar, donde uno a lo mejor entiende su trabajo como..., no sé (...), poner el hombro; ${ }^{4}$ pero a la vez también enfrentar como..., como lo que está arriba; lo que está arriba oprime. Así que eso, yo pienso como en la palabra resistencia, resistentes así, como material de resistencia. $\left(\mathrm{D}_{3}, 33\right)$

La constitución de los cuerpos de los trabajadores y las trabajadoras como cuerpos resistentes es experimentada por estos como una tensión constante. Esta tensión genera

${ }^{4}$ Apoyar, colaborar. 
una contradicción existencial persistente que pone en juego su permanencia en los programas para los que trabajan. Desde ahí, estos trabajadores y trabajadoras comienzan a cuestionarse las razones por las que continúan soportando y empujando el cuerpo de la política de infancia existente, a pesar que este pareciera oponerse infatigablemente al cambio. Este cuestionamiento se soluciona de al menos dos maneras: la renuncia o la transformación cotidiana del espacio de trabajo.

Desde allí uno entra en contradicción con tus principios éticos. Hay una cuestión que viene así, como de preguntarte a ti misma: ¿cómo chucha estoy soportando esta hueá leste trabajol, por qué lo hago?, ¿lo hago solamente una cuestión económica?... Bueno, mejor entonces trabajo en otra cosa. Pero también viene con un compromiso al decir no sabes que yo quiero, de alguna manera, estar dentro de esto, porque es lo que me permite empezar a cambiar las intervenciones; decirle [a las familias]: «Pucha ${ }^{6}$ mira, oye, mira esta otra alternativa, no nos quedemos con lo que es la orientación». (D1, 40)

En este sentido, cuando los trabajadores y trabajadoras deciden continuar en sus trabajos, lo hacen con la condición de no cumplir plenamente con lo que establece la política de protección a la infancia existente, siendo su principal guía la política de protección a la infancia imaginada. El objetivo de esto es continuar reconociendo tanto los derechos de los NNA para quienes trabajan, así como también sus propios derechos y su labor. Esto se convertirá en el motor de su movimiento y lo que mantendrá sus cuerpos como cuerpos resistentes.

[Cuando] en estos trabajos del corte más social (...) no hay un reconocimiento, ni hay una valorización respecto de lo que tú estás haciendo diariamente, en realidad ahí viene el desdén, viene la desgana, viene muchas veces decir: «Bueno y pa que estoy haciendo esta cuestión [trabajo], si esta empresa no me valora». Bueno, entonces ahí uno también entra y dice: «Bueno, lo hago entonces ¿por quién?, ¿lo hago por mí?... Ya, pero también lo hago por las personas que estoy viendo». Ese es como el motor. (D15, 11)

En suma, según los relatos de las personas entrevistadas, uno de los cuerpos que los interpela afectivamente es la política de protección a la infancia que estos imaginan. Esta política ideal - universal, sistémica y con enfoque de derecho- otorga capacidades a los cuerpos de los trabajadores y las trabajadoras que la política existente coopta (Massumi,

\footnotetext{
${ }^{5}$ Chilenismo. En este caso denota desprecio.

${ }^{6}$ Chilenismo. Denota contrariedad, molestia.
} 
1995). Estas capacidades están relacionadas con la rehumanización de los cuerpos y con la constitución de estos como cuerpos resistentes, llenos de vida. Aquí, la resistencia es entendida como una tensión de fuerzas que empuja, a la vez que presiona u oprime. Esta tensión genera contradicciones que tendrán como salidas posibles la renuncia o la transformación del trabajo cotidiano, siendo esto último el motor de estos cuerpos resistentes.

\section{Discusión}

Los hallazgos de la presente investigación sugieren que los cuerpos de los trabajadores y las trabajadoras de la política de protección a la infancia en Chile han sido deshumanizados, aterrorizados y despojados de su energía mediante las interpelaciones afectivas cotidianas del trabajo administrativo y de la política misma. Esto indica que la incorporación del NMP en los servicios sociales, no solo ha implicado una transformación en las maneras concretas de gestionar y ejecutar la política de infancia, sino que también ha sido un intento de apropiación, captura y subordinación afectiva de su fuerza de trabajo (Gómez-Villar, 2016). Como plantea Cena (2015), la «managerialización», como forma de regulación neoliberal, hace cuerpo en determinadas prácticas en pos de un particular régimen de sensibilidad que posibilita la reproducción del neoliberalismo a largo plazo. Dicho régimen de sensibilidad se ha centrado en el establecimiento de acciones de gestión financiera que aseguran la eficiencia en el uso de los recursos públicos. El foco en acciones centradas en la eficiencia y eficacia económica ha permitido la construcción de una política de infancia que tecnifica la labor de sus trabajadores y trabajadoras, no considerando la complejidad de una intervención integral y con enfoque de derechos (Pavez et al., 2019). Esta situación pone en tensión a los trabajadores y las trabajadoras, en tanto les exige enfocarse y cumplir con una serie de labores administrativas que merman el tiempo que le dedican a la intervención y que no dan cuenta de los procesos que experimentan junto a los NNA (Fardella-Cisternas et al., 2016).

No obstante, el estudio de los afectos permite develar que también existe la resistencia. Uno de los elementos que la hacen posible son los cuerpos no-humanos imaginados por los trabajadores y trabajadoras (Ahmed, 2017). En efecto, será la política de protección a la infancia imaginada por estos y estas profesionales la que les permita constituirse como agentes dentro de los contextos laborales en los que se encuentran. El reconocimiento de esta experiencia relacional afectiva nos permite darle centralidad a la posición activa de los cuerpos-humanos en un contexto neoliberal y «managerial» del trabajo público, 
develando que estos y estas trabajadoras son actores y actrices capaces posicionar la humanidad como un elemento primordial de su quehacer, en contraposición a la organización oficial del trabajo de la política de protección a la infancia chilena.

De esta manera, los resultados presentados plantean que la subjetividad de las trabajadoras y los trabajadores está atravesada fuertemente por el afecto (Schaefer, 2019). Será en la relación afectiva entre los indicadores de éxito y mecanismos de evaluación de la política en donde los cuerpos de trabajadores y trabajadoras serán interpelados, afectando las maneras en que estos le dan sentido a la política y construyen su subjetividad laboral (Sellar, 2015). Asimismo, a través de la relación afectiva entre los cuerpos humanos y nohumanos es posible iluminar una experiencia laboral alternativa, creativa, agencial y situada (Deleuze \& Guattari, 2015), que no se encuentra contemplada en las prácticas oficiales neoliberales y administrativas de la política de protección a la infancia de Chile.

Este trabajo es, por sobre todo, un esfuerzo por rescatar voces y afectos que han sido escasamente visibilizados en la construcción de la política de infancia, considerándolos como un elemento central en la ejecución de dicha política, así como el lugar en donde se fisura la hegemonía de la NMP y la ideología neoliberal que la constituye (Ahmed, 2017). Lo anterior se hace aún más relevante si consideramos que Chile se encuentra en un proceso de reestructuración esta política. Esta se deriva de una serie de develaciones mediáticas de abusos y negligencias ocurridas dentro de las organizaciones dependientes de Sename en los últimos años. A raíz de esto, en diciembre de 2020, luego de una extensa discusión parlamentaria, se aprobó la ley 21302 que crea el nuevo Servicio Nacional de Protección Especializada a la Niñez y Adolescencia. Acorde con esto, en octubre de 2021 comenzó la instalación de este nuevo servicio — denominado «Mejor niñez»— iniciándose así el proceso de transformación del antiguo Sename. En este contexto, el presente trabajo aporta elementos necesarios para tener en consideración para construir una nueva política de protección a la infancia con enfoque de derechos, situada y con sentido, que apunte al cuidado de los NNA en situación de vulneración de derechos. Para ello, es necesario tener en cuenta tanto la experiencia laboral encarnada de los trabajadores y las trabajadoras que ejecutan esta política como el conocimiento sensible que estos y estas han adquirido a lo largo de su trayectoria profesional en Sename.

Los resultados presentados instan a continuar estudiando los afectos dentro de la política de protección a la infancia, comprendiendo que trabajadores y trabajadoras se constituyen, no solo desde el gobierno de sus racionalidades, sino también desde la experiencia encarnada de las intensidades que se mueven entre cuerpos humanos y no- 
humanos (Gherardi et al., 2018). Respecto a esto, aún queda la tarea de desarrollar metodologías que estén orientadas a encontrar el rastro de los afectos en la construcción de la subjetividad laboral (Knudsen \& Stage, 2015). Lo anterior es un desafío, no solo en términos de los estudios de la política de protección a la infancia, sino que también dentro de los estudios de la psicología social del trabajo en Latinoamérica.

Debido al contexto de pandemia en el que se realizó la investigación, una de las limitaciones de este estudio es que la producción de datos se realizó únicamente de manera virtual. En este sentido, sería interesante profundizar en la experiencia laboral encarnada de los trabajadores y las trabajadoras de la política de infancia de forma presencial. Asimismo, para futuras investigaciones, se sugiere considerar que, si bien en este artículo planteamos que la política de infancia imaginada permite que los trabajadores vuelvan a construir sus cuerpos como humanos, esta rehumanización no es total; tiene partes que siguen estando maquinizadas, creando sujetos fragmentados o quiméricos (Deleuze \& Guattari, 2015). Desde ahí, proponemos profundizar en estas subjetividades múltiples, los afectos que las constituyen y en los efectos que estas tienen en el quehacer laboral cotidiano de los trabajadores y trabajadoras.

\section{Agradecimientos}

Agradecemos al Sindicato Interempresa Nacional de Trabajadores/as Subcontratados del Servicio Nacional de Menores por su apoyo en el proceso de producción de datos de este proyecto. En particular, agradecemos a las personas que participaron de la investigación y que nos abrieron las puertas de sus vidas y trabajos.

\section{Referencias}

Ahmed, S. (2017). La política cultural de las emociones. Universidad Autónoma de México. Albaek, A. U., Kinn, L. G., \& Milde, A. M. (2018). Walking children through a minefield: How professionals experience exploring adverse childhood experiences. Qualitative

Health Research, 28(2), 231-244. https://doi.org/10.1177/1049732317734828 Araujo, K. (2019). Hilos tensados: para leer el Octubre Chileno. Editorial Usach. 
Ashcraft, K. L. (2017). «Submission» to the rule of excellence: Ordinary affect and precarious resistance in the labor of organization and management studies. Organization, 24(1), 36-58. https://doi.org/10.1177/1350508416668188

Bealer, T. (2019). Consider the dementor: Discipline, punishment, and magical citizenship in Harry Potter. Dialogue, 6(3), 36-47.

Cena, R. (2015). Políticas sociales, cuerpos y emociones a principios del siglo XIX en Argentina. Convergencia, 22(69), 213-232. https://doi.org/10.29101/crcs.v22i69.3652

Charmaz, K. (2007). Constructing grounded theory. Sage.

Chen, M. Y. (2012). Animacies. Duke University Press.

Cromby, J. (2012). Feeling the way: Qualitative clinical research and the affective turn. Qualitative Research in Psychology, 9(1), 88-98. https://doi.org/g7tq

Cubillos-Celis, P. (2021). Infancia en Chile 1973-2013: 40 años de tensiones e inflexiones neoliberales. Revista Latinoamericana de Ciencias Sociales, Niñez y Juventud, 19(3), 1-22. https://doi.org/10.116oo/rlcsnj.19.3.4868

Davies, B., \& Gannon, S. (2006). Doing collective biography. Open University Press.

Deleuze, G., \& Guattari, F. (2015). Mil mesetas: capitalismo y esquizofrenia. Pre-Textos.

Denzin, N. K. (2001). The reflexive interview and a performative social science. Qualitative Research, 1(1), 23-46. https://doi.org/10.1177/146879410100100102

Enciso, G. (2015). Una travesía de las emociones al afecto en las prácticas del poliamor [Tesis de Doctorado]. Universitat Autonoma de Barcelona.

Errázuriz, V. (2021). Becoming «Hijas de la Lucha»: Political subjectification, affective intensities, and historical narratives in a Chilean all-girls high school. Theory \& Research in Social Education, 49(1), 27-53. https://doi.org/g7tr

Fardella-Cisternas, C., Sisto, V., Morales, K., Rivera, G., \& Soto, R. (2016). Identidades laborales y ética del trabajo público en tiempos de rendición de cuentas. Psykhe, $25(2), 1-11$.

Fardella-Cisternas, C., García-Meneses, J., Soto-Roy, A., \& Corvalán-Navia, A. (2021). Exacerbados: identidades académicas y la transformación de la educación superior chilena. Quaderns de Psicologia, 23(2), e1602. https://doi.org/g7tv

Flick, U. (2004). Introducción a la investigación cualitativa. Morata.

Flyvbjerg, B. (2011). Case study. En N. Denzin \& Y. Lincoln (Eds.), The Sage handbook of qualitative research (pp. 301-316). Sage.

Foucault, M. (2008). The birth of biopolitics: Lectures at the College de France, 1978-1979. Palgrave Mcmillan. 
Fox, N., \& Alldred, P. (2013). The sexuality-assemblage: Desire, affect, anti-humanism. The Sociological Review 61 (4), 69-789. https://doi.org/10.1111/1467-954X.12075

Gherardi, S., Murgia, A., Belle, E., Miele, F., \& Carreri, A. (2018). Tracking the sociomaterial traces of affect at the crossroads of affect and practice theories. Qualitative Research in Organizations and Management: An International Journal, 14(3), 295-316. https://doi.org/10.1108/QROM-04-2018-1624

Gherardi, S., \& Laasch, O. (2021). Responsible management-as-practice: Mobilizing a posthumanist approach. Journal of Business Ethics. https://doi.org/g7tz

Glinsner, B., Sauer, B., Gaitsch, M., Penz, O., \& Hofbauer, J. (2019). Doing gender in public services: Affective labour of employment agents. Gender, Work \& Organization, 26(7), 983-999. https://doi.org/10.1111/gwao.12263

Gómez-Villar, A. (2016). El abandono: el lugar (des)habitado por las vidas precarias. Athenea Digital, 16(1), 113-136. https://doi.org/10.5565/rev/athenea.1737

Holstein, J., \& Gubrium, J. (1995). The active interview. Sage. https://doi.org/bkg2m9

Hood, C. (1991). A public management for all seasons? Public Administration, 69(1), 3-19. https://doi.org/10.1111/j.1467-9299.1991.tboo779.x

Hunter, S. (2015). Power, politics and the emotions: Impossible governance? Routledge-Cavendish. https://doi.org/10.4324/9780203798041

Jupp, E., Pykett, J., \& Smith, F. M. (2017). Emotional states: Sites and spaces of affective governance. Routledge. https://doi.org/10.4324/9781315579252

Katila, S., Laine, P. M., \& Parkkari, P. (2017). Sociomateriality and affect in institutional work: Constructing the identity of start-up entrepreneurs. Journal of Management Inquiry, 18(3). https://doi.org/10.1177/1056492617743591

Knudsen, B. T., \& Stage, C. (2015). Introduction: Affective methodologies. En B. T. Knudsen, \& C. Stage (Eds.), Affective methodologies (pp. 1-22). Palgrave Macmillan. https://doi.org/10.1057/9781137483195_1

Marques de Mattos, C., \& Dal Castel, V. (2015). Excelência e produtividade: Novos imperativos de gestão no serviço público. Psicologia \& Sociedade, $27(2), 322-331$. https://doi.org/10.1590/1807-03102015v27n2p322

Massumi, B. (1995). The autonomy of affect. Cultural Critique, 13(31), 83-109.

McKenzie, M. (2017). Affect theory and policy mobility: Challenges and possibilities for critical policy research. Critical Studies in Education, 58(2), 187-204. https://doi.org/gh24jx

Nishida, A. (2017). Relating through differences: Disability, affective relationality, and the U.S. public healthcare assemblage. Subjectivity, 10(1), 89-103. https://doi.org/g7t8 
Organisation for Economic Co-operation and Development. (2005). Modernising government: The way forward. https://bit.ly/3d6KGos

Pavez, J. (2018). Significados y prácticas de la precariedad en trabajadores y trabajadoras de un programa social de infancia en la región de Valparaíso. [Tesis de doctorado]. Pontificia Universidad Católica de Valparaíso.

Pavez, J., Herrera-Soto, Y., Molina-Johannes, J., Ortúzar-Flores, H., \& Zamora-Bugueño, C. (2019). El permanente conflicto entre Estado e instituciones colaboradoras a partir de su marco normativo. Athenea Digital, $19(1), 1-24$.

Penz, O., \& Sauer, B. (2019). Neoliberal technologies of social protection: Affects and policy implementation. Critical Policy Studies, 14(3), 339-357. https://doi.org/g7t9

Penz, O., Sauer, B., Gaitsch, M., Hofbauer, J., \& Glinsner, B. (2017). Post-bureaucratic encounters: Affective labour in public employment services. Critical Social Policy, 37(4), 540-561. https://doi.org/10.1177/0261018316681286

Pereira, J. M. M. (2015). Continuidade, ruptura ou reciclagem? Uma análise do programa político do Banco Mundial após o Consenso de Washington. Dados, 58(2), 461-498. https://doi.org/10.1590/00115258201550

Pink, S., Horst, H., Postill, J., Hjorth, L., Lewis, T., \& Tacchi, J. (2016). Etnografía digital. Morata.

Pitton, V. O., \& McKenzie, M. (2020). What moves us also moves policy: The role of affect in mobilizing education policy on sustainability. Journal of Education Policy. https:// doi.org/10.1080/02680939.2020.1852605

Rojas, C. (2018). Afecto y cuidado: pilar de la política social neoliberal. Polis, ${ }_{17}(49)$, 127-149. https://doi.org/10.4067/s0718-65682018000100127

Ruthrof, H. (1997). Semantics and the body. University of Toronto Press.

Schaefer, D. (2019). The evolution of affect theory. Cambridge University Press.

Schöngut-Grollmus, N. (2017). Ensamblajes socio-técnicos para la producción de intervenciones psicosociales en un programa de Servicio Nacional de Menores de Chile. Psicoperspectivas, 16(3), 41-51. https://doi.org/d5jm

Seigworth, G., \& Gregg, M. (2010). An inventory of shimmers. En G. Seigworth, \& M. Gregg (Eds.), The affect theory reader (pp. 1-25). Duke University Press.

Sellar, S. (2015). A feel for numbers: Affect, data and education policy. Critical Studies in Education, 56(1), 131-146. https://doi.org/10.1080/17508487.2015.981198

Servicio Nacional de Menores. (2019). Balance de gestión integral año 2018. Ministerio de Justicia y Derechos Humanos de Chile. https://bit.ly/3rpczaG 
Sisto, V. (2020). Desbordadas/os: rendición de cuentas e intensificación del trabajo en la universidad neoliberal: el caso de Chile. Archivos Analíticos de Políticas Educativas, 28(7), 1-26. https://doi.org/10.14507/epaa.28.4907

Sisto, V., Núñez-Parra, L., \& Rivera-Aguilera, G. (2021). Entre managerialismo y nostalgia: el debate por el futuro de la universidad y el trabajo académico. Quaderns de Psicologia, 23 (2), e1695. https://doi.org/10.5565/rev/qpsicologia.1695

Sisto, V., \& Zelaya, V. (2013). La etnografía de Dispositivos y el estudio de los instrumentos de rendición de cuentas como prácticas. Universitas Psychologica, 12(4), 1345-1354.

Soto, R. A., Mera, J. M., Nuñez, C. G., Sisto, V. M., \& Fardella, C. (2016). Between the effectivity and affectivity: New teachers in times of new public management. Athenea Digital, 16(3), 3-19.

Varas, A., Carrasco, A., Gutiérrez, D., \& Bascuñán, A. (2018). La explotación en el «área social»: una lectura de la precariedad laboral del tercer sector en Chile desde El Capital, de Marx. Izquierdas, (39), 273-298. https://doi.org/g7ve

Vivero-Arriagada, L. (2017). Trabajo Social entre el sentido común, hegemonía y praxis: un análisis basado en Gramsci. Revista Latinoamericana de Ciencias Sociales, Niñez y Juventud, $15(1), 547-563$. 